DOE/ER-00382

\title{
THE HUMAN GENOME INITIATIVE OF THE
}

U.S. DEPARTMENT OF ENERGY Office of Energy Research Office of Health and Environmental Research
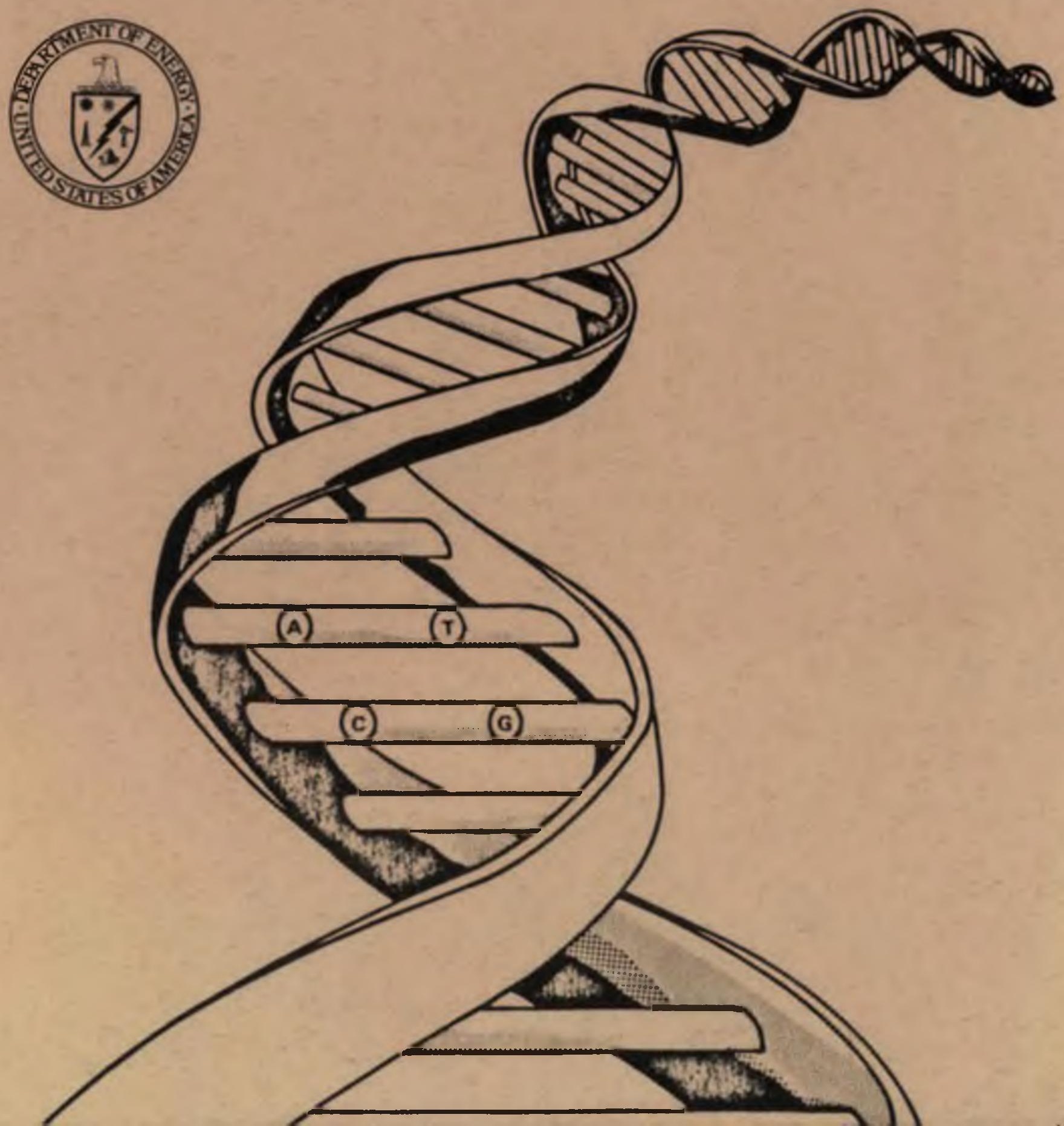


\section{DISCLAIMER}

This report was prepared as an account of work sponsored by an agency of the United States Government. Neither the United States Government nor any agency Thereof, nor any of their employees, makes any warranty, express or implied, or assumes any legal liability or responsibility for the accuracy, completeness, or usefulness of any information, apparatus, product, or process disclosed, or represents that its use would not infringe privately owned rights. Reference herein to any specific commercial product, process, or service by trade name, trademark, manufacturer, or otherwise does not necessarily constitute or imply its endorsement, recommendation, or favoring by the United States Government or any agency thereof. The views and opinions of authors expressed herein do not necessarily state or reflect those of the United States Government or any agency thereof. 


\section{DISCLAIMER}

Portions of this document may be illegible in electronic image products. Images are produced from the best available original document. 
This report has been reproduced directly from the best available copy.

Available from the National Technical Information Service, U. S. Department of Commerce, Springfield, Virginia 22161.

Price: Printed Copy A03

Microfiche A01

Codes are used for pricing all publications. The code is determined by the number of pages in the publication. Information pertaining to the pricing codes can be found in the current issues of the following publications, which are generally available in most libraries: Energy Research Abstracts, (ERA); Government Reports Announcements and Index (GRA and I); Scientific and Technical Abstract Reports (STAR); and publication, NTIS-PR-360 available from (NTIS) at the above address. 


\title{
THE HUMAN GENOME INITIATIVE OF THE DEPARTMENT OF ENERGY
}

\author{
Office of Energy Research \\ Office of Health and Environmental Research \\ Washlngton, D.C. 20545
}

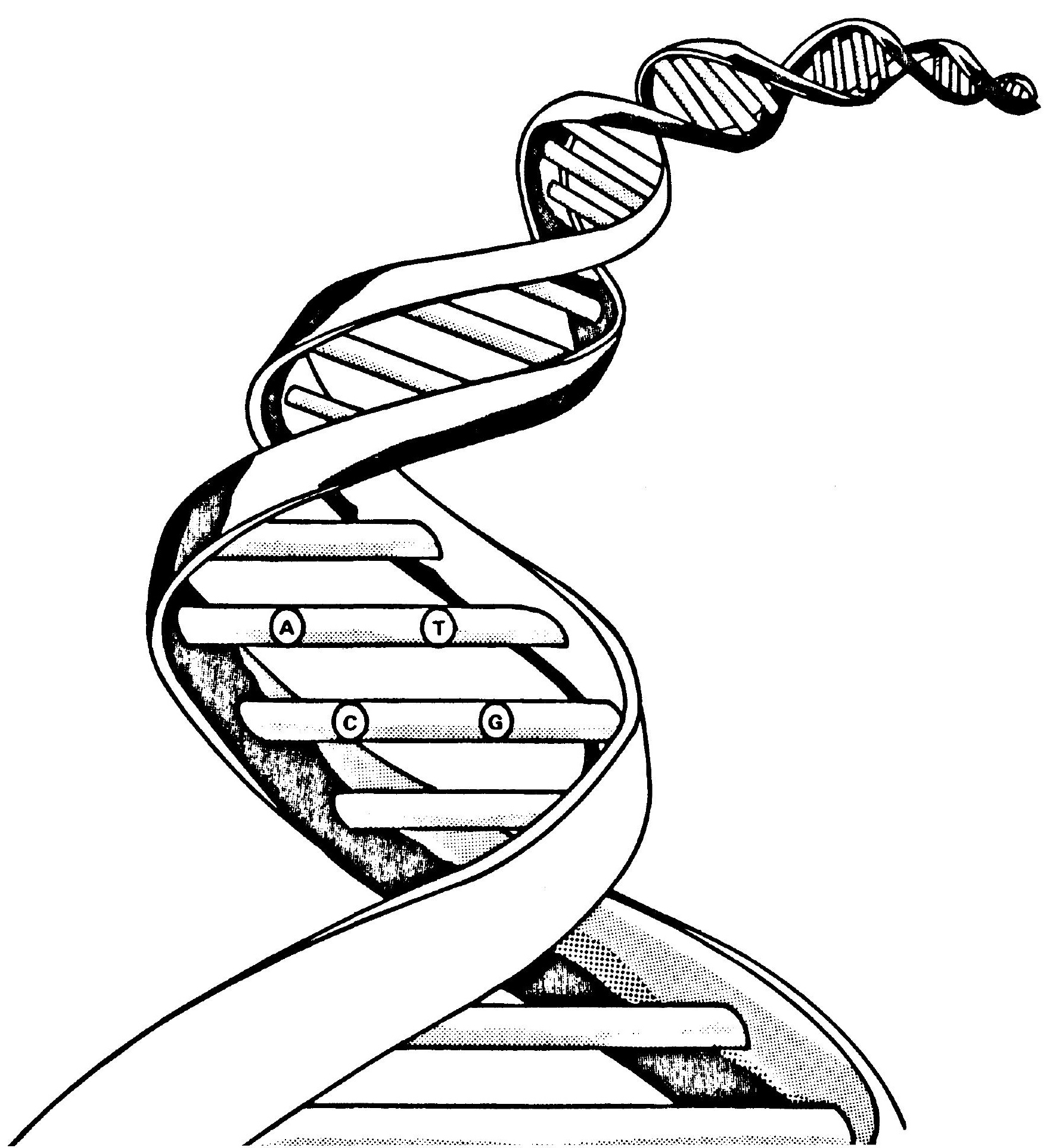


CONTENTS

$\underline{\text { Page }}$

INTRODUCTION

1

A HUMAN GENOME INITIATIVE PRIMER $\ldots \ldots \ldots \ldots \ldots \ldots \ldots \ldots \ldots \ldots \ldots \ldots$

MANDATES OF THE DOE OFFICE OF HEALTH AND ENVIRONMENTAL RESEARCH $\ldots \ldots \ldots 4$

DOE SPONSORED RESEARCH AND TECHNOLOGIES FOR BIOMEDICAL RESEARCH $\ldots \ldots \ldots 5$

ORIGINS OF THE HUMAN GENOME INITIATIVE $\ldots \ldots \ldots \ldots \ldots \ldots \ldots \ldots \ldots \ldots \ldots$

MANAGEMENT AND COORDINATION OF GENOME RESEARCH $\ldots \ldots \ldots \ldots \ldots \ldots \ldots \ldots \ldots$

IMPLEMENTATION OF THE HUMAN GENOME INITIATIVE $\ldots \ldots \ldots \ldots \ldots \ldots \ldots \ldots \ldots$

RECOMMENDATIONS FROM THE APRIL 1987 HERAC REPORT $\ldots \ldots \ldots \ldots \ldots \ldots \ldots \ldots 13$

RECOMMENDATIONS FROM WORKSHOP ON REPOSITORIES, DATA MANAGEMENT AND

QUALITY ASSURANCE FOR THE NATIONAL GENE LIBRARY AND ORDERING PROJECTS . ..15

RECOMMENDATIONS FROM THE WORKSHOP ON DATA MANAGEMENT FOR

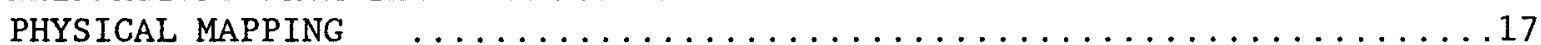

THE 1988 SPECIAL GENOME INITIATIVE REVIEW PANEL $\ldots \ldots \ldots \ldots \ldots \ldots \ldots \ldots$

ABSTRACTS OF HUMAN GENOME INITIATIVE RESEARCH AND DEVELOPMENT PROJECTS . . 19 


\section{INTRODUCTION}

The structural characterization of genes and elucidation of their encoded functions have become a cornerstone of modern health research, biology and biotechnology. A genome program is an organized effort to locate and identify the functions of all the genes of an organism. Beginning with the DOE-sponsored, 1986 human genome workshop at Santa Fe, the value of broadly organized efforts supporting total genome characterization became a subject of intensive study. There is now national recognition that benefits will rapidly accrue from an effective scientific infrastructure for total genome research. In the U.S. genome research is now receiving dedicated funds. Several other nations are implementing genome programs. Supportive infrastructure is being improved through both national and international cooperation. The Human Genome Initiative of the Department of Energy (DOE) is a focused program of Resource and Technology Development, with objectives of speeding and bringing economies to the national human genome effort. This report relates the origins and progress of the Initiative.

\section{THE INITIATION OF DEDICATED HUMAN GENOME RESEARCH}

Pre 1986

March 1986

April 1986

June 1986

July 1986

August 1986

January 1987

April 1987

May 1987

September 1987

March 1988

April 1988

May 1988

September 1988
Genome projects on microbes implemented

The Santa Fe workshop assess human genome project feasibility

A two phase DOE Initiative announced

Cold Spring Harbor Symposium on Homo sapiens

Howard Hughes Medical Institute forum

National Academy of Sciences workshop

Inclusion of human genome funds in the presidential budget

HERAC Report on the DOE Human Genome Initiative

National Institutes of Health genome program announcements

Lawrence Berkeley and Los Alamos National Laboratories designated as DOE Human Genome Initiative research centers

National Research Council report

Congressional Office of Technological Assessment report

Cold Spring Harbor Meeting on progress in genome research

International Human Genome Organization (HUGO) formed. 


\section{A Human Genome Initiative Primer}

Within each of the tissues of the human body a drama of function, cooperation and at times defense is played. The primary actors are macromolecules, the DNAs, RNAs and proteins. Their actions are directed by the estimated 50-100,000 genes encoded on the 24 distinct human chromosomes. The genetic directions drive embryogenesis and the specialized functions of our tissues. Genes are mutable. Most changes within a lifetime pass unnoticed, but some can be carcinogenic. Changes inherited over the generations increase the exciting diversity of humankind, but some cause inherited diseases or weakness towards particular diseases. Understanding the body's dramas and the consequences of mutation is a major goal of modern health research.

Currently only some 1,400 human genes are available for detailed study in the form of identified clones of chromosome fragments. Trying to decipher the actions of 50,000 interacting genetic directors is difficult, and even more so when the identity of most of the genes remains to be established. This has been a severe handicap, one greatly limiting efforts to understand the body's functioning in health and disease. But this handicap is being eliminated as the nation progresses with total genome research. These efforts will greatly speed and bring economies to the understanding of all human genes.

The largest continuous human DNA segment now cloned, physically mapped and sequenced is the $150,000 \mathrm{bp}$ (base pair subunits of DNA) growth hormone region. The whole human genome is more than 20,000 times larger, encoded in 3 billion bp of DNA. This vastly greater entire genome task requires the development of much improved resources and technologies, enhanced interpretive capacities and a coordinated and supportive $R \& D$ infrastructure.

To analyze any segment of the genome in detail, corresponding DNA must be obtained in a pure form. The cloning process fulfills this requirement. Human DNAs are millions of $b p$ in length in the cell. They are first broken into fragments of suitable size. Each fragment is united with helper genes. A recombinant DNA is thus formed which is viable only in special host cells.

cellular DNAs or sorted chromosomes

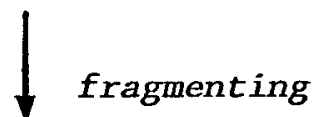

chromosome fragments / / / /

joining with helper genes*

recombinant DNAs $/ * / * / * / * / *$

$\downarrow$ absorbing into special host cells

viable recombinant DNAs in host cells

clones of recombinant DNA $/ *^{/ *} / *^{*} / *^{*} / *^{/ *} / *^{/ *} / * t *^{/ *} / *^{/ *} / *$ 
Each recombinant replicates with or within its host, into a clone with millions of identical members. From the clone, the identical copies of its particular human genome segment are easily purified.

The collection of clones which together represent a whole chromosome or genome is a library. A complete library must have enough clones to both "cover" the chromosomes and cover the DNA breaks introduced by cloning. The shorter the cloned human segments are, the larger the complete library must be. Currently recombinants with human DNA segments up to 40,000 bp long are easily constructed, and cloning systems for 10 fold larger DNAs are being validated. For cloned segments 40,000 bp long, the smaller human chromosomes will each have libraries with several thousand clones, and 500,000 clones will be in the 24 chromosomal libraries combined. Automated systems must be developed for the processing of such huge clone collections.

Within a newly constructed library, much of the physical continuity of human chromosomes is hidden because of the initial DNA breakage step in cloning. Continuity is revealed by ordering the library. Structural analyses of the human segment of each clone are performed. The structural data is used to compare clones, identifying clone pairs having genetically overlapping segments of human DNA. Each initial DNA break in cloning is thus bridged by the genetic overlap of two clones. The clones of the library can then put in an order representing the intact human chromosome.

The ordered 1ibrary has far greater value than the unordered clones. Some larger genes are unavoidably broken during cloning. Their overlapping pieces are adjacent in the ordered library. With the overlaps discounted, the library provides a physical map of the chromosome. The positions of already identified genes serve as known addresses on the map. They are alignment points for coarser genetic maps, which are drawn from human inheritance studies. A newly sought gene can be rapidly found in the ordered library, once the gene's position on a human chromosome is roughly known. The ordered library is the best source of DNA for genome sequencing, because the excess DNA overlaps have been identified and can be omitted.

cellular DNAs or sorted chromosomes

24 libraries of clones

ordered libraries and physical maps of the 24 distinct chromosomes

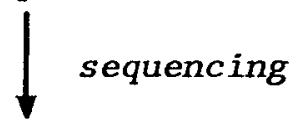

Reference sequence of the human genome

$\downarrow$ interpreting

Greatly enhanced capacities in medicine, health research and biotechnology 
The most detailed chromosome information is obtained through sequencing, which reveals the order of the four subunits of DNA. Until recently, only a few hundred bp were sequenced consecutively, at costs above a dollar per bp. Improving strategies are bringing economies to cloning and sequencing, and more are necessary. Novel methodologies are being developed and they must be implemented and tested. At a target level of one cent per bp, a transition from the current costly sequencing of more important genes to an economical, organized genome sequencing will become feasible.

Obtaining a reference sequence of the human genome is now a national objective. Optimal utilization of the vast genome resource requires improvements in our capacity to manipulate biological databases, to compare DNA sequences and to correlate encoded information with that of other biomedical sources. In the past, genes were first detected through mutations causing inheritable diseases. In the future, genes will be much more rapidly recognized within the DNA sequence. Identified genes are an extraordinary basic resource. With the genes available, strategies for deciphering their actions are easily designed. The encoded human proteins can be produced as pharmaceuticals. Diagnostic reagents to monitor health comprehensively can be assembled.

Our efforts to interpret and understand the genome and the many dramas of the body will long continue, and require contributions from diverse branches of medicine, information sciences and biotechnology. But with a complete genetic directory, humanity will be far better equipped for this exciting challenge.

\section{MANDATES OF THE DOE OFFICE OF HEALTH AND ENVIRONMENTAL RESEARCH (OHER)}

The current roles and responsibilities of OHER are inherited through the Atomic Energy Commission (AEC) and its successor agencies. The Atomic Energy Act of 1946 (P.L. 79-585) provided the initial charter for a comprehensive program of research and development related to the utilization of fissionable and radioactive materials for medical, biological, and health purposes. The Atomic Energy Act of 1954 (P.L. 83-703) further authorized the AEC "to conduct research on the biologic effects of ionizing radiation."

The Energy Reorganization Act of 1974 (P.L. 93-438) provided that responsibilities of the Energy Research and Development Administration (ERDA) shall include "engaging in and supporting environmental, biomedical, physical and safety research related to the development of energy resources and utilization technologies." The Federal Non-nuclear Energy Research and Development Act of 1974 (P.L. 93- 577) authorized ERDA to conduct a comprehensive non-nuclear energy research, development, and demonstration program to include the environmental and social consequences of the various technologies.

The DOE Organization Act of 1977 (P.L. 95-91) mandated the Department "to assure incorporation of national environmental protection goals in the formulation and implementation of energy programs; and to advance the goal of restoring, protecting, and enhancing environmental quality, and assuring public health and safety," and to conduct "a comprehensive program of research and development on the environmental effects of energy technology and program." 
RESOURCES AND TECHNOLOGIES FOR BIOMEDICAL RESEARCH SPONSORED BY THE AEC, ERDA AND DOE

Liquid Scintillation Radiation Detection

Scanning Transmission Electron Microscope

Cyclotron generators for short-lived isotopes

High Flux Isotope Reactor

Positron Emission Tomography

Boron Neutron Capture Radiotherapy

Radioactive pharmaceuticals for diagnosis and therapy

National Synchotron Light Source

Synchrotron angiography

High Flux Beam Reactor

Particle accelerators for structure analysis and radiation therapy

High pressure liquid chromatography

Continuous flow zonal ultracentrifuge

Ames mutagen detection system

Cryogenic preservation of murine embryos

Fluorescence Activated Cell Sorter

National Flow Cytometry Resource

National Gene Library Project

GenBank Data Sequence Repository

Protein Structure Data Bank

Gamma Ray Camera

Advanced computation facilities

Advanced detectors for X-ray crystallography 


\section{ORIGINS OF THE DOE HUMAN GENOME INITIATIVE}

In fulfilling its responsibilites the AEC provided the first major federal support for genetics research. It also sponsored the development of high technology instruments and systems for health and environmental research. An extensive program of DOE sponsored research on genome structure, maintenance, damage and repair continues at universities and national laboratories. In these studies, it became evident that there is variation among individuals in susceptibility to damage from background radiation and environmental chemicals.

A major goal of the program was consequently formulated: the development of capacities to diagnose individual susceptibility to stresses imposed by energy related and environmental factors on the integrity of the genome. A 1984 conference in Alta, Utah was co-sponsored by DOE and the International Commission for Protection Against Environmental Mutagens and Carcinogens. It highlighted growing roles of novel DNA technologies in diagnostics. Their state-of-the-art descriptions are substantially incorporated in the 1986 Congressional Office of Technology Assessment Report "Technologies for Detecting Heritable Mutations in Human Beings". The novel approaches increasingly included gene cloning and sequencing. The isolation of sought genes from libraries of clones has been an integral component of biomedical research for many years. But the one-gene-at-a-time procedures employed are wasteful of scientists' time and research resources.

In 1985, DOE began to consider whether its expertise with high technology projects could be fruitfully applied to achieving a rapid access to all human genes. Programs to identify all genes of several viruses began in the 1960s, and were the birth place of many important chromosomal methodologies. With the advent of molecular cloning techniques in the 1970s, a library of manageable DNA clones could be produced for any species. Genome studies on many viruses, the bacterium Escherichia coli, a yeast and the minute nematode worm were subsequently implemented. With the constituent skills already demonstrated, a human genome program is a task in engineering and program development. However, it is a task on a massive scale which had not previously been considered in biology. A successful implementation would rapidly generate knowledge and products of both scientific and commercial value. The accompanying resource and technology development would bring advances and economies to many ongoing biomedical and biotechnological projects, in addition to advancing particular DOE programs.

To assess the desirability and feasibility of ordering and sequencing clones representing the entire human genome by the year 2000, DOE sponsored in March 1986 an international meeting in Santa Fe, New Mexico. The participating experts concluded with virtual unanimity that this objective was meritorious, obtainable and would be an outstanding achievement in modern biology. Further guidance was sought from the Health and Environmental Research Advisory Committee (HERAC) of the DOE, which provided its April 1987 Report on the Human Genome Initiative.

The HERAC report urged DOE and the Nation to commit to a large, multidisciplinary, technological undertaking to order and sequence the human genome. This effort would first require significant innovation in general 
capability to manipulate DNA, major new analytical methods for ordering and sequencing, theoretical developments in computer science and mathematical biology, and great expansion in our ability to store and manipulate the information and interface it with other large and diverse genetic databases.

The HERAC report further recommends that DOE have a leadership role because of its demonstrated expertise in managing complex and long-term multidisciplinary projects, involving both the development of new technologies and coordination of efforts of industries, universities, and its own laboratories. The report noted that the mission of OHER to understand the health effects of radiation and other by-products of energy production requires fundamental knowledge of the effects of damage to the genome, and that mission has led to the

initiation and support of a number of research and technological developments which are closely linked to the human genome mapping and sequencing project: DOE computer and data management expertise initiated and sustains the GenBank DNA sequence repository; the chromosome sorting capacities essential to the Genome Initiative were developed and are maintained at DOE laboratories; within the associated National Gene Library Project, libraries of clones representing single human chromosomes are produced for the research community. Thus, the Initiative is a natural outgrowth of ongoing DOE-supported research.

The DOE responded to the Santa Fe meeting and HERAC reports by implementing several pilot projects as the beginning of a two phase program. In Phase I a broad array of technologies and resources are being developed which will have continuing value. The primary biological products are libraries of ordered cloned DNA fragments representing the 24 distinct human chromosomes. In Phase II, the clones will be decoded into a reference sequence of the human genome.

Several government agencies, scientific societies and industrial organizations have studied associated policy and strategy issues and have presented their recommendations. The most prominent are those of HERAC, the National Research Council (NRC) and the Congressional office of Technological Assessment (OTA). The latter are broadly in accordance with the scientific recommendations of the earlier HERAC recommendations. They further recommend that several non-human species be included in the national effort, and that genetic mapping complementary to physical mapping be simultaneously pursued. An international group of eminent scientists has formed the Human Genome Organization (HUGO) to advise and help coordinate world wide efforts.

The ongoing DOE Initiative of resource and technology development is focused on the human genome, but many products of the program will also be applicable to other species and advance programs of other agencies. It complements, but does not include, the genetic mapping pursued by medical geneticists which requires human family studies and roughly locates disease genes. This genetic mapping, plant and animal model studies and related infrastructure are substantially supported by the Department of Agriculture, National Institutes of Health, National Science Foundation and the Howard Hughes Medical

Foundation. 


\section{MANAGEMENT AND COORDINATION OF GENOME RESEARCH}

The highly multidisciplinary and long-term character of the genome research is novel to biological research. An infrastructure connecting biomedical research, technology development, computer sciences, data repositories, physical repositories and supporting agencies has thus become essential.

Agencies most actively supporting genome research formally meet under auspices of the White House Office of Scientific and Technology Policy, but this management structure may be modified by forthcoming congressional legislation. Processes for the coordination of human genome research and technical activities of the DOE and the NIH are specified in a 1988 Memorandum of Understanding. A joint advisory committee, with members drawn from the HERAC and the NIH Program Advisory Committee on the Human Genome, reports to both agencies. There is also extensive informal interagency contact between program administrators. Panels convened by DOE or NIH to review genome research proposals are attended by representatives of both agencies.

Within the DOE, the management structure recommended by HERAC is utilized.

- Policy, strategy and scientific guidance is provided by HERAC.

- A separate scientific board(s) of peers reviews research proposals, including those originating within DOE laboratories. Both prospective and retrospective evaluations are utilized.

- OHER administers the awards, cooperates with all concerned agencies and organizations, organizes periodic workshops, and responds to the needs of the developing program.

- A Human Cenome Steering Committee assists OHER by: providing overall coordination of DOE-funded genome research; spreading novel genome technologies; insuring proper management of data and samples; optimizing overlap among research groups; interfacing to national and international efforts; and helping to communicate the program to the press and public. Members of the committee are drawn from participants in Initiative research. Task groups are formed to address specific issues.

To obtain further guidance and report to the public, workshops and meetings are arranged: in January 1987, a DOE workshop on "Exploring the Role of Robotics and Automation in Decoding the Human Cenome" was held; a June meeting brought together participants in DOE pilot projects and related specialists to discuss program priorities and balance; an August meeting co-sponsored by DOE and NIH discussed "Repositories, Data Management, and Quality Assurance Needs for the National Gene Library and Genome Ordering Projects;" in September DOE sponsored the workshop for professional science writers entitled "Biotechnology and the Human Genome: Innovations and Impacts;" a May 1988 workshop jointly planned by DOE, NIH and the HHMI considered outstanding problems concerning "Data Management for Physical Mapping." This latter workshop developed recommendations on data and DNA probe accessibility, database structure standardization, standardization of nomenclature and issues to be addressed further. Genome sessions have been organized by DOE for the XVI International Congress of Genetics and the 1989 Meeting of the American Association for the Advancement of Science. 


\title{
1988 COORDINATION OF GENOME RESEARCH
}

White House Office of Scientific and Technology Policy (OSTP)

1

Federal Coordinating Council on

Scientific \& Engineering Technologies (FCCSET)

Standing Committee on the Life Sciences

The Subcommittee on the Human Genome

I I I I I I

Department of National Howard Hughes National Department of

Energy Institutes Medical Science Agriculture

of Health Foundation Foundation

\section{DOE HUMAN GENOME INITIATIVE MANAGEMENT AND COORDINATION}

\author{
HERAC \\ Asst. Sec. for \\ other \\ Energy Research / agencies \\ review panels \\ Office of Health and \\ Environmental Research \\ Human Genome I Steering Committee \\ 1 \\ Projects at universities, national laboratories and industry
}

excerpt from the 1987 OTA report "Mapping Our Genes-The Genome Projects:

How Big, How Fast?"

Most issues that need to be addressed regarding genome projects are variations on the problem of the commons: how to create and maintain resources of use to all. It can be difficult to develop goods useful to all if each individual has no direct incentive to pay for them and only a few are adversely affected.

The core issue concerning genome projects is resource allocation. What priority should be given to funding databases, materials repositories, genetic map projects, and development of new technologies? Should genome projects have precedence over other projects important to biological and biomedical research? These projects will benefit the entire biomedical research community, and ultimately the Nation and the world, but their funding must be drawn from the same agencies that funding support basic research. Funding for genome projects will thus be taken from agencies that support research on neuroscience, cancer, immunology, and many other promising and rapidly moving fields. 
Pre 1986

Fluorescence Activated Cell Sorter developed

National Flow Cytometry Resource for sorting chromosomes

National Gene Library Project (NGLP) producing mono-chromosomal libraries

GenBank DNA sequence repository established

Management actions

Santa Fe Meeting

Human Genome Initiative announced

Pilot projects pursued at national laboratories:

computer modeling and optimization of library ordering strategies database management support

advanced instrumentation and vectors for ordering and sequencing artificial intelligence analysis of sequence data

isolation of centromere, telomere and chromosome specific clones

Inter-agency coordination, workshops

HERAC Report on the DOE Human Genome Initiative

Los Alamos and Lawrence Berkeley National

Laboratories are designated Genome Centers

Special Research Grant Program (SPRG) Notice

Peer-review panel for SPRG proposals

1988 research awards

Human Genome Steering Comm. formed

$\underline{\text { Research in progress }}$

Resource development:

Phase II NGLP cosmids with 40,000 bp insert

Yeast artificial chromosomes

Normalized cDNA libraries

Low copy number repetitive DNAs

ATCC repository pilot project

Synthetic nucleases

Novel mapping instrumentation

Physical mapping:

Macro-restriction mapping of chromosomes 21 and 5

Library ordering for chromosomes 16, 19

$5,11,17,21$, and $\mathrm{X}$ initiated

Sequencing technology development:

Multiplex, single molecule strategies, fluorescent tags, oligomer hybridization, automated DNA preparation, automated autoradiograph decoding

Informatics :

Human Genome Information System development

Dedicated sequence comparison chips 


\section{DOE HUMAN GENOME INITIATIVE RESEARCH IMPLEMENTATION}

Prior to the Santa Fe workshop, there were already several DOE programs providing or maintaining genome research resources for the scientific community. These include the National Gene Library project, the isolation of DNA markers for specific chromosomes/chromosome regions, and the development and maintenance of the GenBank DNA sequence repository. Under phase I of the Gene Library project, chromosomes purified by FACS (fluorescence activated cell sorting) are the source of DNA for constructing libraries of clones representing each of the 24 distinct human chromosomes. The Phase I libraries with $9,000 \mathrm{bp}$ inserts are available from the American Type Culture Collection (ATCC) repository. Single copy DNAs are readily isolated from such libraries; a set of 100 single copy clones for chromosome 21 has been deposited with the ATCC. Centromere and telomere specific DNA probes have also been isolated. The construction of Phase II libraries with 40,000 bp inserts began in 1987. These libraries are important resources for both physical mapping and the medical geneticist's mapping programs as well.

After receiving the recommendations from the Santa Fe workshop, several other pilot projects were initiated. These included novel sequencing technologies, optimization of ordering strategies by computer simulations, artificial

intelligence interpretation of sequence data, initiation of clone ordering for chromosomes 16 and 19, and the initiation of macro-restriction mapping of chromosome 21. Institutions participating were Harvard U., Columbia U., the Genetics Institute (Boston) and the DOE laboratories at Berkeley, Brookhaven, Livermore, Oak Ridge and Richland. Broadly valuable resources and technologies have flowed from these projects.

Multiplex DNA sequencing strategies are being implemented. They provide for significant increases in speed and decreases in costs by pooling many DNA samples at an early processing stage. Information from the multiple samples is recovered after many common intermediate steps by sequential interrogations of the co-resident DNA patterns. Longevity of the patterns under the multiple interrogations has been validated. One system has been licensed for further commercial development and marketing.

A collaboration headed by $C$. Smith and C. Cantor of Columbia University is producing the first DNA restriction map of an entire human chromosome, chromosome 21. This chromosome encodes many genes of recognized importance in human genetic diseases. Their pioneering technique of pulsed field gel electrophoresis (PFE) is crucial to this system of macro-restriction mapping, which achieves physical mapping without any possibility of cloning artifacts.

An advanced control system for PFE and its variants has been developed. Facile microprocessor control and modification of the electric field configuration is achieved. Thus protocols producing desired DNA fragment separations can be rapidly tested and optimized.

DOE computer scientists have constructed mathematical models of alternative ordering strategies and are establishing optimal parameters. In the chromosome 19 library ordering project, a commercial DNA sequencer has been modified to facilitate generation of restriction fragment "fingerprints" of clones. With the use of fluorescent DNA labels, multiple samples are pooled 
and co-processed. The throughput of the analysis system is so high, that the preliminary purification of recombinant DNA from clones is currently the rate limiting factor of the total library ordering.

One recommendation emerging from the August 1987 workshop on repositories and quality control concerned the chromosome sources. In the past, easily grown tumor cell have been commonly used chromosome sources. But for the Human Genome Initiative the preferred source is normal cells, representing a genome of a studied pedigree. A genome among the family pedigrees available from the Center for the Study of Human Polymorphisms (CEPH) was recommended. In response a set of rodent host cell lines, each a host for one of the 24 human chromosome, is now being constructed. They will be a source of human mono-chromosomal DNAs. These "CEPH" hybrids will be made available through the American Type Culture Collection repository as they are constructed and validated.

Early experience in the pilot projects quickly showed data management within the laboratory can become laborious and time consuming. The scale and diversity of materials utilized in genome research is far beyond that encountered in prior experience, with attendant recording burdens. For project participants without sufficient data management experience, DOE specialists visit and provide assistance. Software has been designed to serve as an "electronic notebook" facilitating simple entry and display of data, while providing a database format for latter processing and future transfers within and between laboratories. The DOE ESNET (Energy Sciences Computer Network) is being expanded and its computation and rapid data transfer capacities will be available to the Genome Initiative.

When specific genome funds were dedicated in 1987, the DOE Special Research Grant Program Notice on the Human Genome Initiative was published. A special review panel meet in January 1988 to evaluate some 50 proposals received. Of those funded, 12 awards are to universities, two awards are to DOE scientists one award is to a private laboratory, and one award is to the American Type Culture Collection. Several of the awards are for improvements to, or novel development and testing of, sequencing technologies. The award to the ATCC is for a pilot project, to improve capacities of repositories to manage the vastly increased flux of clones being generated by the genome projects. The abstracts of all DOE Human Genome Initiative related research are provided below.

The HERAC recommendations for Initiative expenditures were developed to meet a goal of completing Phases $I$ and II by the year 2,000. This recommendation for funding by all agencies included FY_88 expenditures of $\$ 20 \mathrm{M}, \$ 40 \mathrm{M}$ in FY_89 and increases of $\$ 40 \mathrm{M}$ per year to a plateau at $\$ 200 \mathrm{M}$ per year in 1993 . The National Research Council report also recommended new funding for the project with increases over a period of three years to the $\$ 200 \mathrm{M}$ per year level. The OTA report projected that $\$ 660 \mathrm{M}$ would be required for the first five years of Phase I, and provided detailed estimates for various aspects of the project. In FY 87 DOE funded $\$ 5 M$ of pilot research projects and the FY 88 funding is at a level of just under \$11M. The FY 89 request is for $\$ 18 \mathrm{M}$. The DOE effort is being complemented by that of $\mathrm{NIH}$, beginning in FY_88. NIH identified funds specifically for new research on complex genomes at the level of $\$ 18 \mathrm{M}$ and is requesting $\$ 28 \mathrm{M}$ for $\mathrm{FY} \_89$. 


\section{The April 1987 Recommendations of the HERAC REPORT on the HUMAN GENOME INITIATIVE}

1. DOE should fund a major new initiative whose goal is to provide the methods and tools which will lead to an understanding of the human genome. Funding should start in fiscal year 1989 at $\$ 40$ million and increase over a five year period to reach a level of $\$ 200$ million per year. Appendix A provides details.

2. The early goals (first 5 to 7 years) of this program should be to:

a) Make a physical map of the human genome. A physical map consists of a complete set of segments of the DNA, arranged in order.

b) Locate genes and other markers on the map.

c) Produce and distribute cloned DNA sequences and other materials needed for using and improving the physical map.

d) Develop new techniques and improve existing methods for large-scale DNA mapping and sequencing (including applications of automation and robotics).

e) Develop new methods for characterizing and locating genes; both computational and cloning techniques are needed.

f) Establish computer facilities, and develop computer data bases for the storage, retrieval and dissemination of cloning, mapping, and sequence information (including cross-references to other relevant data bases). Improve and invent algorithms for analyzing DNA sequences, including methods for identifying coding regions, predicting protein structures and functions, and identifying genetic regulatory sites.

3) The major long-term goal is to obtain a base sequence for each of 24 reference human chromosomes, and to make DNA sequencing technology readily available to search for disease-related variations and to make biological comparisons. The improvements in technology listed in Recommendation 2 are necessary to attain this goal.

4) Work on these goals should take place in the National Laboratories, in universities and in industry. Both prospective and retrospective peer review should be used. Cooperation and collaboration among all groups is essential; in particular, all new map and sequence information must be placed promptly in a designated data base. Clones and cell lines must be made available for distribution to other qualified investigators.

5) Two scientific panels should be established immediately. One would develop policy, define overall strategy, and provide continuing oversight. The other would provide scientific review of proposals and programs for their technical merit and feasibility. The initial phase of the program should consist primarily of technological development in the areas of construction of large scale maps, automation, sequencing and the determination and analysis of sequence data. Because of the highly creative nature of this beginning phase, it is essential that the effort be widely distributed. The project should involve single-investigator-initiated proposals as well as multidisciplinary 
consortia that bring together the development of instrumentation and software, as well as biotechnology.

6) DOE should encourage wide collaboration at the scientific and managerial levels for the human genome project. Cooperation is needed with other agencies within the U.S. and with other countries throughout the world. Results should be open and in the public domain, within the constraints of technology transfer and the promotion of industrial involvements. Information transfer should be emphasized among the cooperating scientists, the scientific community and the public at large.

Human Genome Subcommittee

Ignacio Tinocco, Chairman

$\mathrm{U}$. of California, Berkeley

George Cahill

Howard Hughes Medical Center

Charles Cantor

Columbia University

Thomas Caskey

Baylor College of Medicine

Renato Dulbecco

Salk Institute

Dean L. Engelhardt

Enzo Biochemicals, Inc.

Leroy Hood

California Inst. of Tech.
Leonard S. Lerman

Genetics Inst. (Boston MA)

Mortimer L. Mendelsohn

Lawrence Livermore Nat. Lab.

Robert L. Shinsheimer

$U$. of California, Berkeley

Temple Smith

Harvard Univeristy

Dieter Soil

Yale University

Gary Stormo

University of Colorado

Raymond L. White

University of Utah Med. Center 
RECOMMENDATIONS from the WORKSHOP On REPOSITORIES, DATA MANAGEMENT, and QUALITY ASSURANCE for the NATIONAL GENE LIBRARY and GENOME ORDERING PROJECTS

The 26-27 August 1987 meeting was held in Pleasanton, CA and co-sponsored by the Department of Energy and the National Institutes of Health.

\section{THE NATIONAL GENE LIBRARY RESOURCES}

1. Funding should be made available to establish a set of stable mono-chromosomal hybrids for each of the human chromosomes.

2. A repository should be established for such hybrids.

3. A data base should be established for both the libraries in the repository and those still maintained by the originator.

\section{PHYSICAL MAPPING}

1. Establish a repository of chromosome-specific DNA probes for mapping the large genome fragments by pulsed-field gel electrophoresis.

2. Establish sets of chromosome-specific linking probes (e.g., Not I or $\underline{\text { Sfi }} \underline{I}$ based) for linking the large fragments together.

\section{PHYSICAL REPOSITORY}

1. Examine ways to automate the verification procedures for received material to minimize the associated costs.

2. Expand the repository to include the physical output (i.e., contigs, clones and probes) that will be derived from the chromosome ordering efforts.

\section{RESEARCH NEEDS GENERATED BY THE LIBRARY AND ORDERING PROJECTS}

1. A mechanism should be established to insure that current recipients of government funding make any hybrid cell lines and probes available that are derived from that funding.

2. Journals should take a stronger position in accepting publications on probes and libraries. Acceptance should be contingent upon deposition of the reported materials in a repository.

3. Any new hybrid cell lines established should have the human donor material available in another form e.g., as fibroblast or lymphoblast cultures. If at all possible, only use hybrids for which normal cells or DNA from the donor is available.

4. A mono-chromosomal hybrid panel should be established from family kindreds, even if only two generations (i.e., parents and children) are available. Perhaps the CEPH kindreds would be an appropriate source of material.

5. Use of lymphoblast cells for Phase II libraries should be avoided. Rearrangements in these lines are thought to occur a high frequency.

6. Establish a repository and data base for all hybrid cell lines.

7. Only contigs above a minimum size should be stored in the repository. No consensus was reached on minimum size requirements but suggestions ranged from 100 to $300 \mathrm{~kb}$. Only a $1 \mathrm{X}$ contig coverage should be stored.

8. Any ordered DNA segments to be placed in the repository should be coupled to the genetic map, i.e., have either an RFLP or a gene assigned to it.

9. Southern blots of large fragments from pulsed field electrophoresis separations should be mass produced and stored in a repository for other users. This could be a commercial venture.

10. Dot blots of each contig could also be mass produced and stored in a repository. This should decrease the demand on the repository to store and supply the original clones. 
11. Establish a normalized human cDNA library for each chromosome. These would be especially useful for map closure. Consensus was not reached on tissue of origin of the cDNAs.

12. A contract should be considered to support a pilot project for the repository and data base for the ordering efforts. The ordered library of Escherichia coli established by Kohara and co-workers might serve as test material. The goals would be to develop costs and clone management expertise as a prelude to the creation of a larger repository.

\section{ACCURACY AND QUALITY ASSURANCE}

1. Additional funding should be provided for increased quality control of the Phase II effort of the National Gene Library Project. Most of this effort would be required in characterization of the libraries to insure purity, and the funding would support a necessary additional person at both Los Alamos and Lawrence Livermore National Laboratories.

2. Journals should play a major role in assuring quality control by increasing the stringency of their acceptance criteria for probes and libraries. Proper and sufficient characterization information should be provided.

3. A committee to oversee the global issues related to the national ordering effort should meet frequently to minimize redundancies and insure cooperation.

\section{DATA MANAGEMENT}

1. A data base for restriction fragment data on cosmid clones and large fragments should be established.

2. A standard nomenclature should be developed for the informatics aspects of the genome ordering effort.

3. A smaller workshop should be conducted in the near future to deal specifically with the problems of intra-laboratory data management and inter-laboratory data exchange.

\section{PANELISTS}
A.V. Carrano
J. F. Leighton
P. de Jong
E. W. Branscomb
M.A. van Dilla
B. Gladhill
H. Mohrenweiser
J . Gray
Lawrence Livermore National Laboratory (workshop hosts)
D. Magglot
W. Niermann
American Type Culture Collection
C. R. Cantor, C. Smith
Columbia University
G. Cahil1
Howard Hughes Medical Institute
J. Courteau

L. L. Deaven C. E. Hildebrand W. Goad

Los Alamos National Laboratory

R. Mortimer

Lawrence Berkeley Laboratory

B. Harrison M. Guyer R. Levinson National Institutes of Health
B. J. Barnhart
Department of Energy
K. K. Kidd
Yale University

M. Stodolsky

Argonne National Laboratory

J. Schlinder J.D. Miller

K. M. McKenney

National Bureau of Standards 


\section{SUMMARY of RECOMMENDATIONS from the WORKSHOP On DATA MANAGEMENT for PHYSICAL MAPPING}

The May 12-13, 1988 meeting was held in Bethesda. MD and co-sponsored by the Department of Energy, Howard Hughes Medical Foundation and National Institutes of Health.

- Solving problems of nomenclature is fundamental to addressing data management issues. Efforts to address these problems should begin soon. Two specific issues are DNA probe names and locus names (currently assigned D numbers). Recommendations for addressing these issues include the following:

1. A set of naming conventions designed to assure that clones are assigned unique names, which can be handled by computers, should be developed.

2. A series of workshops and meetings to deal with nomenclature issues should be held.

3. Journal editors should be encouraged to adopt and enforce human nomenclature conventions.

- A panel of well characterized reference markers, identified by DNA clones which are freely available and distributed throughout the human genome, would facilitate mapping efforts. Appropriate committees of the Human Gene Mapping Workshops should be encouraged to designate a set of reference markers which could be made available through the Human Genetic Probe Repository.

- Policies to encourage exchange and accessibility of probes should be developed.

- A working group to coordinate physical mapping studies and develop ways to represent each type of map in relational way should be organized. The group might evolve into a committee to maintain physical mapping standards.

- Convenient and widely adaptable software for managing physical mapping data should be developed. The software should permit investigators to organize and manage data within their own laboratories, to exchange data with other laboratories, and to locate other databases with relevant information.

- Facile transfer of data is essential to the genome project. A computer network with rapid file transfer capacity is needed. The computer network would be most useful if it also had mail and bulletin board features .

- The need for and organization of a comprehensive database for genetic and physical maps of humans and model organisms should be considered. To best address the needs of the scientific community, a mapping database should be relational. Future discussions should address the organization of a mapping database, the functions a mapping database should support, as well as the attributes of the data to be stored.

- Existing databases as well as databases to be established in the future should contain pointers which would refer investigators to relevant information in other databases. Computer-assisted queries in databases or in a separate system would be useful. 
Panelists

Tom Shows, Chairman

Roswell Park Memorial Institute

Dennis Benson

National Library of Medicine

Elbert Branscomb

Lawrence Livermore National Lab.

Doug Brutlag

Stanford U. School of Medicine

Walter Goad

Los Alamos National Laboratory

Kenneth Kidd

Yale University

Mark Lathrop

University of Utah
Donna Maglott

American Type Culture Collection

Jacob Maize1

Ferderick Cancer Rsch. Center

Thomas Marr

Los Alamos National Laboratory

Daniel Masys

National Library of Medicine

David Schlessinger

Washington University

Cassandra Smith

Columbia University

Robert Waterston

Washington University

\section{REVIEW PANEL for the 1988 DOE GENOME INITIATIVE RESEARCH PROPOSALS}

\begin{abstract}
Anthony Carrano
Lawrence Livermore

National Lab.

George Church

Harvard Medical Sch.

David Cox

$\mathrm{U}$. of $\mathrm{CA}, \mathrm{SF}$

Ronald Davis

Stanford University

Richard M. Fine

Columbia University

David E. Housman

Mass. Inst. of Tech.
\end{abstract}

Burke H. Judd

Nat. Inst. Env. Health Sci. Kansas State Univ.

Jakob Maise1

Frederick Cancer Rsch Fac.

Sean McGlynn

Louisiana State University

Keith McKinney

Nat. Bureau of Standards

Jurg Ott

Columbia University

John Rosenburg

$U$. of Pittsburg
Donald Roufa

Cassandra Smith

Columbia University

John Sulston

MRC Lab. of Mo1. Biol.

George Trainor

E.I. Du Pont Company

Edward Yeung

Iowa State University

Philip Youderian

Univ. Southern CA, 


\section{ABSTRACTS OF DOE HUMAN GENOME INITIATIVE RESEARCH PROJECTS}

These abstracts are primarily excerpts from the "Research in Progress: FY 1988" report of the DOE Office of Health and Environmental Research. Some additional recently started projects are also included. Many of the projects were initiated in June or July 1988, and their cited funding does not represent continuing yearly levels.

\section{Resource development}

\section{Human Recombinant DNA Library}

Deaven, L.L.

Life Sciences Division, Los Alamos National Laboratory, Los Alamos, NM. 87545. 505-667-3114;FTS 843-3114

The goal of the National Laboratory Gene Library Project is the production of chromosome-specific human gene libraries and their distribution to the scientific community for studies of the molecular biology of genes and chromosomes, and for the study and diagnosis of genetic disease. This is a cooperative project involving the flow sorting and molecular cloning expertise at Los Alamos National Laboratory (LANL) and Lawrence Livermore National Laboratory (LLNL). The specific aim of this phase of the project is the production of complete digest ( $4 \mathrm{~kb}$ average insert size) libraries from each of the human chromosomal types purified by flow sorting. The bacteriophage vector is Charon 21A, which has both Eco R1 and Hind III insertion sites accommodating human DNA fragments 0 to $9.1 \mathrm{~kb}$ in size. Each laboratory will produce a complete set of chromosome-specific libraries, LANL with Eco R1 and LLNL with Hind III. In this way human DNA fragments missing in one library (because restriction sites are too far apart) are likely to be found in the other library. Library purity will depend upon the quality of the sorting procedure, which is about 908 for most chromosomal types isolated from cultured fibroblasts. These libraries are constructed in a conservative way with well-conceived techniques, to minimize production time. With their small insert size, they will be of major use in studies and diagnosis of genetic disease. Beyond this phase, we will move to the construction of libraries with large inserts (about $20 \mathrm{~kb}$ ) in more advanced, recently developed bacteriophage vectors or in cosmids (about $40 \mathrm{~kb}$ ), and with characteristics better suited to basic studies of gene structure and function. In addition to libraries of normal human DNA sequences, we will clone DNA from rearranged (aberrant) chromosomes useful in studies of chromosome structure, genetic disease and neoplasia.

\section{Human Genome Information Resource Fickett, J.W. , $\$ 366,000$ Theoretical Division, Los Alamos National Laboratory, Los Alamos, NM, 87545. 505-667-6690;FTS $843-6690$}

Coordinating and advancing the mapping and sequencing of the human genome will depend heavily on a central computer-based information resource for which GenBank is the closest existing model. Management, long-term refinement of strategies, and coherent organization of the results of the project require sophisticated information management on a scale hundreds of times larger than GenBank. The objective is to develop the technology for a central information 
resource that can provide all participating scientists facile access via computer network. The approach will be to define and implement a new structure for the GenBank data base, and to provide it with a network interface. The result will be an initial small-scale resource that can support chromosome mapping and strategy planning, and thus serve as a test bed for modeling needs and solutions for a full-scale human genome information resource.

\section{Genome Organization and Function}

Moyzis, R.K.

Life Sciences Division, Los Alamos National Laboratory, Los Alamos, NM. 87545. 505-667-2746;FTS $843-2746$

The long-range objective of this project is to determine the molecular mechanisms by which higher organisms organize and express their genetic information. Applications of these investigations include development of novel approaches for detection of human genetic diseases, and measuring effects of low-level ionizing radiation and/or carcinogen exposure. A combination of biochemical, biophysical, and recombinant DNA techniques are used to identify, isolate, and determine the roles of DNA sequences involved in long-range genomic order. Current efforts focus on determining (1) the organization and function of human repetitive DNA sequences and (2) the factors regulating organization and expression of metallothionein (MT) and related genes. Investigations in the past year include (1) demonstration that hypermethylated genes can be early replicating, which suggests there is no causal relationship between gene activity, hypermethylation, and replication timing; (2) demonstration of coordinate amplification of MT-I and MT-II genes in cadmium-resistant cells; and (3) isolation (for the first time) of human chromosome-specific repetitive DNA sequences. Future studies will focus on spatial organization in the MT multigene cluster, and definition and isolation of functional repetitive DNA regions. Defining the mechanisms responsible for organizing the mammalian genome, and the genetic and nonmutational alterations accompanying abnormal phenotypic change are important aspects of defining the effects of energy-related technologies. Determining the genetic variability in these mechanisms provides a rational basis for establishing thresholds for toxic substances, for making valid cross-species extrapolations, and for identifying individuals at risk.

\section{Optimizing Procedures for a Human Genome Repository}

Nierman, W.C.

American Type Culture Collection, 12301 Parklawn Dr., Rockville, MD, 20852. $301-231-5559$

Recently, there has been intense interest in initiating an organized project to determine the physical map of the human genome and ultimately to determine the complete DNA sequence. DOE-sponsored physical mapping projects to generate overlapping clones of chromosomes 16 and 19 are in progress at the Los Alamos and Lawrence Livermore Laboratories, respectively, and a project to determine a large-scale restriction map of chromosome 21 is ongoing at Columbia University. For the overlapping clones to be useful for additional research and sequencing, each must be readily available to the research community. As demonstrated by the success of the NIH Repository of Human DNA Probes and Libraries, the most efficient way for these clones to be made available is 
for them to be placed in a public repository. Estimates of the number of clones required for a physical mapping project to give coverage of the entire genome range from 90,000 for minimally overlapping cosmid clones to perhaps 600,000 . The establishment and operation of a repository for this number of clones is far beyond the experience of any institute. In order to explore what methods would be necessary to operate a repository on this scale, it is proposed that a pilot repository be established. Approximately 1300 overlapping lambda clones from the genome of Saccharomyces cerevisiae, as well as several thousand clones from the DOE human chromosome 16 and 19 physical mapping projects, will be accessioned to the pilot repository over a two-year period. The operation of this pilot repository will be directed toward testing and optimizing procedures for such functions as verifying clone structure, clone preservation, storage, and distribution, and data management. Analysis of the data from this pilot repository should permit a better evaluation of the effort and costs required to establish and operate a repository conducted on the scale of the entire human genome project.

\section{Collection of Low Copy Number Repeats for Use as Probes in Human DNA} Schmid, C.W.

Department of Chemistry, University of California, Davis, CA, 95616. $916-752-3003$

Clones of interspersed repeats present in low copy numbers in human DNA would be a valuable collection of reagents for plans to map the genome. These clones will be isolated by a procedure of subtractive hybridization of known human repeated sequences from a clone library constructed from renatured DNA. Following this subtraction, the remaining cloned repeats will be characterized according to a number of properties that will make them useful as hybridization probes in human mapping studies. We will determine their copy number, interspersion pattern, sequence divergence with regard to optimal hybridization condition, and evolutionary conservation with regard to detecting human DNA present in hybrid cell lines. It is likely that the collection of clones will be a very useful resource in global mapping efforts.

\section{National Gene Library}

Van Dilla, M.A.

$\$ 790,000$

Biomedical Sciences Division, Lawrence Livermore National Laboratory, Livermore, CA, 94550. 415-422-5662;FTS 532-5662

The goal of the National Laboratory Gene Library Project is the production of chromosome-specific human gene libraries and their distribution to the scientific community for studies of the molecular biology of genes and chromosomes, and for the study and diagnosis of genetic disease. This is a cooperative project involving flow sorting and molecular cloning expertise at Los Alamos National Laboratory (LANL) and Lawrence Livermore National Laboratory (LLNL). The aim of Phase I is to produce complete digest ( $4 \mathrm{~kb}$ nominal average insert size) libraries from each of the human chromosomal types purified by flow sorting. The bacteriophage vector is Charon 21A, which was both Eco R1 and Hind III insertion sites accommodating human DNA fragments 0 to $9.1 \mathrm{~kb}$ in size. Each laboratory produces a complete set of chromosome-specific libraries, LANL with Eco RI and LLNL with Hind III. Human DNA fragments missing in one library (because restriction sites are too far apart) are likely to be found in the other library. Library purity will 
depend upon quality of the sorting procedure which is over 90 to 958 for good chromosome preparations and favorably placed peaks in the flow karyotype to considerably poorer purity for less than optimal preparations and unfavorably placed peaks. Libraries are constructed in a conservative way with established techniques, to minimize production time. With their small insert size, they are of major use in studies and diagnosis of genetic disease. Phase II involves constructing libraries with large inserts (about $20 \mathrm{~kb}$ ) in more advanced, recently developed bacteriophage vectors or in cosmids (about 40 $\mathrm{kb}$ ). Large insert libraries will be suited to basic studies of gene structure and function, organization of genes on chromosomes, and ordering of cloned sequences. LANL has been exploring the large insert phage vector Charon 35, and LLNL has been exploring cosmid cloning.

Normalized cDNA Libraries

Weissman, S.M.

Department of Human Genetics, Yale University, New Haven, CT. 06510

$\$ 183,000$ $203-785-2677$

The project objective is to develop and demonstrate methods for preparing normalized cDNA libraries and using them for gene mapping and mutation detection. We will develop methods for rapidly cloning the DNA spanning the break points in chromosome deletions and rearrangements, even when these rearrangements are present in a heterozygous state in the cellular source of the DNA. The specific aims are: (1) to prepare cDNA libraries containing sequences from either the $5^{\prime} 200$ to 300 bases or the $3^{\prime} 400$ to 500 bases of mRNA species present in various cellular sources; (2) to "normalize" the cDNA libraries in (1) so that they contain an approximately equal number of copies of CDNA for each mRNA expressed in the original cell source; (3) to use the libraries of (2) to isolate a set of full-length "normalized" cDNA clones in an expression vector; (4) to use OFAGE blots and chromosomal library DNA to map the members of the libraries in (1), (2), and (3) to specific chromosomal regions; (5) to develop methods for forced expression of genes so that, in combination with the methods of the preceding specific aims, we can construct a generalized normalized cDNA library containing equal representation of most mRNA transcribed during development; (6) to develop approaches so that the index cDNA library of (5) can be used to rapidly identify tissue specific and chromosomal region specific cDNA clones, and to detect mutations affecting the genes encoding these cDNAs; and (7) to develop methods for rapid mapping of deletion end points suitable for systematic mapping of gene function in mammals. 


\section{Physical Mapping}

Computational Analysis and Support for Extensive Physical Mapping of Genomes Goad, W.

Health, Safety, and Environment Division, Los Alamos National Laboratory, Los Alamos, NM, 87545. 505-667-1600;FTS 843-1600

The very large DNA molecules into which eukaryotic genomes are organized (containing up to several times $10^{8}$ base pairs) must be highly fragmented for cloning and analysis. The arrangement of fragments in the original molecule can be determined if they can be linked to one another by overlaps, either among themselves or among themselves and other fragments generated for the purpose. The overlapping fragments may be generated in various ways, and various kinds of information can be used to characterize fragments' nucleotide sequences to establish overlap. The project objectives are (1) to develop and apply computational tools to study the conditions and effort needed for conducting various strategies, and (2) to directly support mapping efforts as they develop. Activities are (1) to develop a general way of representing the data such that all of the possible kinds of information are accessible in a common data base; (2) to develop algorithms to solve the map from the data; (3) to generate simulated data for promising strategies, to study the effects of clone library structure, to organize repetitive elements, and to study uncertainties and errors of the outcome; (4) to analyze the simulated data to evaluate experimental design and probable courses of mapping efforts; and (5) to support the developing experimental program through data collection into a common data base, providing general access to computational tools and to the results of analysis.

\section{Mapping Genomes Using Cluster Labels}

Hainfeld, J.F.

Biology Department, Brookhaven National Laboratory, Upton, Long Island, NY, 11973. 516-282-3372;FTS 666-3372

The high-resolution Scanning Transmission Electron Microscope (STEM) at Brookhaven National Laboratory (BNL) was used to gather unique structural data that could play a vital role in integrating information from molecular biologists and from light and conventional electron microscopic (CEM) studies of chromosomes. Heavy atom clusters (with 8/angstrom/ diameter cores visible in the STEM) have been developed into specific markers that can be covalently attached to specific sites on molecules; these provide localization at 5 to 30 times better resolution than with other techniques. Complementary DNA to known sequences of interest can be labeled with heavy atom clusters (by incorporating phosphorothioated bases), resulting in a high-resolution structural probe to map the sites of important genes or sequences. DNA binding proteins can be specifically labeled, and it may be possible to label drugs or other small molecules that interact directly with the DNA. A new antibody label recently was synthesized with a gold cluster that is 5 to 10 times smaller than other electron microscope antibody labels. This new probe could better delineate the localization of sequences or proteins for which antibodies exist or will be developed. We will attempt to determine the feasibility of sequencing DNA directly with the microscope. 
Robust Detailed Mapping of the Human Genome

Lerman, L.S.

$\$ 254,000$

School of Science, Massachusetts Institute of Technology, Cambridge, MA, 02139 $617-253-4705$

This project involves a novel approach to physical mapping that may be valuable in constructing maps of the human genome. The method is radically different in principle from others in current use or discussion, but simple to execute. It does not depend on sites of restriction endonuclease cleavage and it does not require cloning of the region to be mapped. The method recognizes as markers regions of DNA that are lower in thermal stability than neighboring regions. It displays intervals between these markers and intervals between the markers and cloned probes as distinct features in patterns from two-dimensional denaturing gradient gels. The samples from which the patterns are generated consist of random fragments of genomic DNA produced by shear or another non-site-specific agent. The patterns provide information for rapid ordering or probes. The maps are easily set into correspondence with restriction maps, but unlike the latter, they are insensitive to single-base variations. The maps can reveal regions of substantial methylation without serious loss of the usual map information. Because each of the various markers that respond to a probe are fairly precisely characterized, the system would be useful in mapping deletions, insertions, translocations, and so forth.

Novel Methods for Physical Mapping of the Human Genome Applied to the Long Arm of Chromosome 5

McClelland, M.

Department of Biochemistry and Molecular Biology, University of Chicago,

Chicago, IL, 60637. 312-702-9470

Our goal is to assess an array of techniques for the megabase physical mapping of human chromosomes. We will apply these techniques to a problem of manageable size, namely the mapping of a 25 megabase (Mb) region of chromosome 5 that is deleted in radiation and mutagen-induced leukemia. The techniques to be assessed include: (1) three methods for the production of unique sequence clones from the region of interest; (2) novel methods for the production and separation of multimegabase DNA fragments; (3) methods for the production of "physical linking clones" that contain rare restriction sites; and (4) application of these methods and available resources (chromosome 5 jumping library, assigned probes, somatic cell hybrids, and chromosome libraries) to map the region of interest.

Techniques for Determining the Physical Structure of Entire Human Chromosomes Smith, C.L. $\$ 538,000$

Department of Genetics and Development, Columbia University, New York, NY, 10032. 212-305-4145

The project objective is to construct a macrorestriction map of human chromosome 21. Large DNA fragments of this chromosome will be generated by restriction nuclease digestion, separated by pulsed field gel electrophoresis, and analyzed by probing with cloned DNA assigned to chromosome 21 and with specialized linking clones made from flow-sorted chromosome 21. Cytogenetic analysis and partial digestions will provide supplementary and confirmatory information. New techniques will be developed 
for rapid physical mapping (i.e., separations of even larger DNAs than currently feasible, and improved methods to purify and work with individual large DNA fragments).

New Approaches to DNA Mapping: Synthetic Endonucleases Sutherland, B.M.

Biology Department, Brookhaven National Laboratory, Upton, Long Island, NY, 11973. $516-282-3293 ;$ FTS 666-3293

Recognition and mapping of functionally important DNA regions (e.g., regulatory and coding regions, initiation sequences) can be greatly facilitated by specific DNA cleavage at such sites. Synthetic endonucleases able to cleave at regions of functional importance will be created by coupling DNA site-specific binding proteins via linker arms to light-activatable cleaving moieties. Specific binding function is provided by the DNA binding protein, and cleavage activity is provided by the activatable cleaving molecules. A prototype system of Rose Bengal coupled via a hexanoic acid linker to a DNA lesion site-specific monoclonal antibody will be developed for other specific DNA binding proteins, including T7 RNA polymerase and mammalian transcription initiation factors. Additional cleaving groups, linkers, and coupling procedures will be developed to optimize reaction with DNA binding proteins of differing surface groups and reactivities. The specificity and efficiency of binding and cleavage of each synthetic endonuclease will be using a new electronic imaging system. We will evaluate their use in mapping, cloning, and sequencing human DNA.

Mapping instrumentaion

Electronic Autofluorography of Two-Dimensional Macromolecular Distribution Davidson, J.B. $\$ 137,000$

Instrumentation and Controls Division, Oak Ridge National Laboratory, Oak Ridge, TN, 37831. 615-574-5599; FTS 624-5599

Two approaches to DNA sequencing will be developed: (1) a modification of the EIDEC system for electronic imaging of two-dimensional gels and (2) an on-line method in which the movement of the fragments provides the scanning motion for four detectors built into the drifting apparatus. The EIDEC imaging detector converts radioactivity to light by means of an interposed scintillator. Resulting light is intensified by a sensitive electro-optical tube, and the image is stored as positive charge on the target of a camera tube. After exposure, the integrated image is transferręd to a çmputer memory for analysis. We have detected and quantitated ${ }_{\mathrm{H}},{ }^{14}{ }_{\mathrm{C}},{ }^{35} \mathrm{~S}$, and ${ }^{32} \mathrm{P}$ in gels and on nitrocellulose. Single ${ }^{32} \mathrm{P}$ betas have been observed. For application to rapid sequencing, we will modify the system to work directly from the drifting apparatus. This will eliminate the necessity for transferring the gel to a backing, drying, and so forth. When drifting is completed, a short exposure of the order of a few minutes will transfer the image to a computer programmed to read the sequence. As drifting proceeds snapshots of the gel will be possible, to determine optimum drifting time and to measure mobilities. In the second approach a detector will be placed over each track of the sequencing gel. The fragments will pass under four defining slits. The outputs of the four detectors will be combined in a microcomputer where the bases will be determined and displayed at each interval of the 
drifting time. Both approaches will be studied for possible application to a system in which many streams of robotically produced fragments are fed into a continuously operating sequenator.

Quantitation in Electrophoresis Based on Lasers

Yeung, E.S.

Environmental Sciences Program, Ames Laboratory, Ames, IA, 50011.

$\$ 137,000$ $515-294-8062$

This project will develop a novel laser-based imaging technique for quantitation in electrophoresis. No stains are required, thus improving on reliabiity, convenience, and speed of processing. The scanning technique uses no mechanical parts, thus adding to the positional accuracy (resolution) of the measurement. This technique will be used to study damage in biological materials for assessing the environmental impacts of energy utilization. The research is based on indirect fluorometry and acoustooptic imaging. In indirect fluorometry, a fluorescing ion is used to elute the sample, producing a large fluorescence background signal throughout the gel. When one of the components of the samples appears, the fluorescing ion is displaced, and a lower fluorescence signal will be observed. Since electrophoresis is based on charged species, electroneutrality requires a one-to-one displacement of the fluorescing ion. This negative signal potentially allows nonfluorescing species to be detected with the high sensitivity normally associated with fluorescing species only, and without staining. The response should be uniform and predictable because it is derived from the same fluorescing ion. Preliminary results on gels indicate that indirect fluorometry is feasible for monitoring proteins. Reliable scanning of the gel can be accomplished by acoustooptic scanners that deflect the laser beam. We have recently shown that two scanners orthogonal to each other can give two-dimensional maps of atoms in the millisecond time scale. Adaptation of this imaging technique to gels should be possible. The scan can be repeated many times per second to further enhance signal-to-noise ratios.

\section{Library ordering projects}

Molecular Mapping of Chromosomes 17 and X: High Resolution Genetic Maps of Chromosomes 17 and $\mathrm{X}$

Barker, D.F.

Medical Informatics, University of Utah, Salt Lake City, UT 84108.

$\$ 222,000$

$801-581-5070$

The strategy for constructing a set of overlapping clones and a very high density map involves the initial use of a moderately dense genetic map to define a series of starting points for island expansion. The initial genetic map will also define specific gaps with an insufficient density of DNA markers. We will attempt to bridge these gaps with a combined genetic and physical mapping approach, avoiding the bulk of the massive screening efforts that would be required to find polymorphic markers in such gaps at random. Our preliminary goal is the construction of a complete set of overlapping clones for an entire chromosome. Production of a very high density genetic map will follow by producing a complete chromosomal restriction map and developing appropriately spaced clones into polymorphic markers. 
Chromosome-Specific Physical Mapping

Carrano, A.V.

Biomedical Sciences Division, Lawrence Livermore National Laboratory,

Livermore, CA, 94550. 415-422-5698; FTS 532-5698

The project objective is to create a linear physical map of human chromosome 19. Chromosome 19 DNA is obtained from flow-sorted chromosomes and hamster-human hybrid cells containing only this human chromosome. The physical map will be arranged as an overlapping set of DNA fragments contained in recombinant DNA vectors and spanning the entire chromosome. The order of these fragments relative to each other will be derived. Our approach is to couple a random ordering strategy, using cosmids with large-scale restriction fragment mapping (using transverse field electrophoresis). Unique cosmid vectors are tailored to achieve maximum clonability, ease of insert isolation, and transcription of insert ends for rapid walking. Random ordering strategies are automated so that the sizes of cosmid-derived restriction fragments can be quantified during the electrophoretic separation, using fluorescent markers. Both chromosome-19-specific and genomic NOT1 linking libraries are being created in newly derived plasmid vectors. They will be used to order the NOTl restriction fragments obtained from transverse field electrophoresis. In addition, total genomic jumping libraries will be made as an aid to map closure. Once the physical map is established, it will be coupled to the known genetic map of the chromosome, thus establishing the colinearity of the two maps and providing a direct correlation between physical and recombination distances for any set of markers. Comparative genetics of a cluster of DNA repair genes on this chromosome should help unravel the DNA repair deficiency syndromes in humans. Chromosome 19 is also the location of several other genes of interest, including a cluster of genes associated with lipoprotein transport.

\section{Mapping and Ordered Cloning of the Human $X$ Chromosome Caskey, T. $\$ 231,000$ Institute of Molecular Genetics, Baylor College of Medicine, Houston, TX. 77030. $713-799-4773$}

The project objective is to isolate a complete set of overlapping DNA clones comprising the human $X$ chromosome. Our initial aim is the construction of a detailed map of the chromosome using pulsed-field gel electrophoresis (PFGE) and somatic cell hybrid mapping with available probes. Additional clones from the terminal half of the long arm of the $X$ will be isolated using DNA from a cell line containing Xq24-Xqter as the only human material in a rodent cell background. Conventional and novel vectors and techniques will be used to saturate the region and to determine overlapping clones. This region will provide a prototype for development of methods for additional segments of the chromosome. Somatic cell hybrids containing other portions of the X will be created using HPRT and introduced dominant selectable markers in experiments, allowing chromosome fragments to be transferred to rodent cell backgrounds. These hybrids will serve as source material for the isolation of region-specific DNA probes, using a novel approach that combines the polymerase chain reaction with selection for human specific repeat DNAs. Probes isolated in this manner will be used to screen lambda, cosmid, or yeast vector libraries in order to isolate human DNA from the region(s) of interest. A major goal is to develop yeast cloning vectors capable of retaining very long insert sequences (on the order of $1 \mathrm{Mbp}$ ), and an efficient 
means of introducing such fragments into yeast, thus simplifying the overall aim by reducing the number of clones genome map $X$ order library necessary. Finally, clones from regions of the $\mathrm{X}$ will be characterized to identify overlapping clusters of clones, using a variety of techniques and the assistance of data base management. Overlapping clusters will then be linked into stretches of clones encompassing the entire long and short arms of the $\mathrm{X}$ chromosome.

Technology Development for Automated Cosmid Mapping of the Human Genome Evans, G.A.

The Salk Institute for Biological Sciences, San Diego, CA, 92138. 619-453-4100

The project objective is to further develop cosmids that will facilitate cloning, chromosome walking, and DNA sequencing. We propose a novel and directed strategy for generating ordered cosmid clones. The use of a solid-phase RNA polymerase-directed synthesis of RNA probes on immobilized cosmid DNAs is part of the system. A major goal is to increasingly implement automation procedures, with the ultimate aim of automatically generating ordered clones representing the human genome. Chromosome 16 and the chromosome region 1lq21-11ter will be analyzed during the developmental program.

Human Genome Chromosome 16

Hildebrand, C.E.

Life Sciences Division, Los Alamos National Laboratory, Los Alamos, NM, 87545. 505-667-2746;FTS 843-2746

The project objective is to construct physical maps of human chromosomes. We will develop a physical map of human chromosome 16 that will integrate restriction enzyme sites, restriction fragment length polymorphic markers, identified gene sequences, and the cytogenetic map. Human chromosome 16 contains numerous genes, gene families, and markers of human genetic disease. Pulsed field gel electrophoresis will be applied to produce a restriction map using infrequently cutting restriction enzymes applied to flow-sorted chromosome 16 DNA or to DNA from somatic cell hybrids with only human chromosome 16 or subregions of it. A complementary approach will use chromosome-specific or subregion-specific large insert libraries produced in phage and/or cosmid vectors. Overlapping clones will be ordered and made available as a resource for further characterization. New technologies for approaching the physical mapping problem will be developed and implemented. Extensive collaborations will be pursued (1) to identify and implement the optimal physical mapping processes identified by simulations and computational analyses of existing and proposed ordering strategies, (2) to develop informational resources to compile physical maps, (3) to improve the acquisition and analysis of physical mapping data, and (4) to identify needs for advanced engineering and technology in mapping and sequencing efforts. A significant result will be information and physical (probes) resources for monitoring mutations and interindividual differences at chromosome 16 loci. 
Ordering Libraires with Human Inserts Maintained by Yeast Artificial Chromosome Vectors

Mortimer, R.K., Esposito, M. $\$ 50,000$ Biology Division, Lawrence Berkeley Laboratory, 1 Cyclotron Rd., Berkeley, CA, 94720. $415-642-4131$

We are developing a physical map and ordered library of the human genome that evantually will be employed for DNA sequencing. The top down approach is being used to physically map the complex human genome by developing yeast artificial chromosome (YAC) libraries of total genomic DNA. This approach was selected because it can cope with the large size and the presence of repeated DNA sequences in the genomes typical of higher eukaryotes. The total genome YAC libraries are being probed by colony hybridization with single copy genes probes and RFLP markers already assigned to particular human chromosomes. The YACS will thus be sorted into chromosome specific libraries. Each 1ibrary will be futher probed with single copy genes and RFLP markers, to obtain a preliminary ordering of each library. Chromosome-specific YACs will also be screened by hybridization in situ to the cognate metaphase chromosomes to coarsely localize the positions of their human DNA inserts. Two methods will be employed to refine the ordering. Yeast genetic crosses will be performed with YACs carrying selective markers, such that the frequency of selected recombinants increases with the extent of genetic overlap of their human inserts. Thus ordering data will be achieved through rapid methodologies based on yeast genetics. A partial physical mapping of YACs will provide an independent data set for ordering the chromosome specific YAC libraries.

\section{Sequencing technologies}

Genomic Sequence Comparisons

Church, G.M.

Department of Genetics, Harvard University, Boston, MA, 02115. 617-732-7562

The project objective is to implement new ideas for rapid, precise, and interpretable DNA sequencing. The approach consists of (1) multiplex sequencing, (2) subtractive sequencing, (3) comparisons between species, and (4) automation of filter hybridization, sequence data reading, and clone walking. Results include: (1) a full test of multiplexing, demonstrating nine reprobings without signal losses and sequencing 80 random oligonucleotides similar to those of the subtractive approach; and (2) a preliminary digital analysis of several sequence films. Expected results include nearly complete sequences for two bacterial genomes ( $10 \mathrm{Mbp}$ total), estimates of the background levels of cross hybridizations in the subtractive and walking strategies, and a determination of the parameters that can minimize such effects. 
Sequencing of Megabase Plus DNA by Hybridization: Method Development Crkvenjakov $R$.

Genome Structure Unit, Center for Genetic Engineering, 11000 Belgrade, Yugoslavia. Telephone 38-11-491-391

Recently, we have formlated the theory of a new method of DNA sequencing, that differs in principle from those already known. It is based on hybridization of all possible oligonucleotides of known 8 mer sequence to clones from a library that allows sufficient representation of sequenced DNA, chromosome or chromosomes. The method requires an industrial plant for its implementation, since approximately 85000 individual hybridizations need to be performed to obtain a sequence. It is the first of this kind in molecular biology research, since it cannot be scaled down to individual research laboratories. Our theoretical considerations indicate that the method might be competitive over existing or methods being developed based on existing principles in both cost and time effectiveness for sequencing DNAs approaching genome size. This application covers experimental testing of all aspects of the method that can be performed in a laboratory. It includes optimization of hybridization with respect to both oligonucleotide probes and cloned DNA, optimization of probe labeling, optimization of library cloning and management procedures, measuring the actual error rate in data acquisition and computer stimulation of data processing and sequence assembly for DNA of a size of a yeast genome. Also, we propose to locate and resequence a known DNA fragment of 2000 base pairs using 2000 probes and hybridizations in an actual yeast genomic library containing approximately 85,000 clones. The aim of the proposal is to provide data for decision whether to proceed to a pilot plant stage.

* This project is being supported under terms of a USA-Yugoslavia Scientific and Technological Cooperation Treaty, with Yugoslavia providing the majority of the funds.

Rapid Preparation of DNA for Automated Sequencing Dunn, J.J.; Studier, F.W. $\$ 122,000$ Biology Department, Brookhaven National Laboratory, Upton, Long Island, NY. 11973. 516-282-2123;FTS 666-2123

Sequencing the human genome is likely to proceed from an ordered set of perhaps 200,000 cosmids, each containing a fragment of human DNA as large as $40 \mathrm{kbp}$. High-capacity automated procedures for determining and handling nucleotide sequences once the appropriate set of DNA samples has been prepared from each cosmid are being developed. However, the process of reducing individual cosmids to a set of DNA samples ready for sequencing is costly and labor-intensive. We will develop an integrated system that will enable a single technician (or an automated work station) to produce thousands of such samples daily, enough raw material for generating a megabase of primary sequence information a day. Priming sites for sequencing by the enzymatic, chain-termination method will be inserted at random sites within the human DNA portion of each cosmid by a selectable transposon, thereby eliminating subcloning steps. Procedures for rapid isolation of these cosmid derivatives will be based on the ability of bacteriophage T7 to replicate and package plasmids that carry appropriate signals, or on the great selectivity of T7 RNA polymerase for its own promoters. Mutants of T7 DNA polymerase having superior properties for sequencing reactions will also be sought. 
Scanning-Tunneling Microscopy

Ferrell, T.L.; Warmack, R.J.

Health and Safety Research Division, Oak Ridge National Laboratory, Oak Ridge, TN, 37831. 615-574-6214; FTS 624-6214;615-574-6215;FTS 624-6215

This project includes operation and continued development of scanning-tunneling microscopes for basic research in physics related to health and environmental problems. A scanning-tunneling electron microscope with single-atom resolution is used to image atomic structure on surfaces, to alter atomic positions, and to probe the dynamical phenomena caused by collective electron motion and motion of ions. Development includes extending capabilities to a wider range of materials, to identification of atomic species, and to studies of biological samples. Methodologies are developed for atom-by-atom studies of biological molecules important in understanding the human genome and in providing other applications in surface science.

Improved Detection Methods Applied to Multiplex Sequencing and Cosmid Walking of Chromosomes 17 and 5

Gesteland, R.F.; Harris, J.; White, R.H. $\$ 368,000$

Department of Human Genetics, University of Utah, Salt Lake City, UT, 84108. $801-581-5190$

The primary effort is to further develop multiplex sequencing, wherein 20 to perhaps a hundred DNA samples can be processed and sequenced in a batch to greatly improve sequencing efficiency. Fragments to be sequenced are cloned into a set of unique vectors each with a special identifier sequence that distinguishes it from the others. A mixture of one of each vector is prepared and sequenced in bulk and the mixed pattern is resolved into each separate sequence by sequential rounds of hybridization with the identifier probes. The probes can be radioactive so that the signal is detected by exposure of $\mathrm{X}$-ray films. Alternatively, fluorescent probes will be developed so that coincident detection of multiple signals can be resolved and detected with an array detector that we will develop. A second effort is the implementation of a novel library ordering strategy for chromosomes 17 and 5. It utilizes hopping from one cloned fragment to the next adjacent piece by use of seeker vectors that allow directional assembly and recovery of adjacent pieces. 
Fluorescence-Detected DNA Sequencing

Haugland, R.P.

$\$ 167,000$

Molecular Probes Inc., 4849 Pitchford Ave., Eugene, OR, 97402. 503-344-3007

Nucleotide analogs that can function as reporter probes when incorporated into polynucleotides are of significant utility in many biochemical procedures and techniques that use recombinant DNA. They are also useful for sequencing DNA. Sequencing of the human genome will require extraordinary effort and major developments in methodology for use of reporter groups that will give one-to-one correspondence with the desired DNA sequence information. It is therefore important to develop ultrasensitive and specific sequencing technology. This application is directed toward major improvements in what appears to be the most promising approach, fluorescence-detected DNA sequencing. The project objective is to develop nucleotides suitably modified with bifluorophores and other novel fluorescent dyes and to provide these to laboratories that are developing fluorescent DNA sequencing methods. This will include Los Alamos National Laboratory and other federally funded laboratories that are developing fluorescent DNA sequencing strategies.

Research will begin with one of the bases (probably thymidine because it has been the primary base used in the biotin system by Ward), using several of the potential fluorophores. This will permit some initial evaluation of the sensitivity of the excitation and fluorescence detection while appropriate derivatives of the other three bases are synthesized. We believe that initial fluorescent derivatives of all four nucleotide triphosphates can be prepared within the first year.

\section{Characterization and Modification of Phage T7 DNA Polymerase for Use in DNA Sequencing \\ Richardson, $C$. \\ Department of Biological Chemistry, Harvard College, Boston, MA, 02115. $617-732-1864$}

The project objective is to understand in molecular terms the complex process by which T7 DNA polymerase and its accessory proteins faithfully copy a DNA template. This will provide us with a reasonable approach to modifying these proteins by chemical and genetic procedures to increase their usefulness with DNA sequence analysis. Specific goals are (1) to improve the yield and purity of the T7 gene 5 protein/thioredoxin complex by coexpressing the genes for both proteins in the same cell and improving the purification procedures; (2) to understand the molecular mechanisms by which thioredoxin confers processivity on the polymerization reaction by examining the interaction of the proteins with each other and with well-defined primer-templates; (3) to elucidate the precise mechanisms by which active oxygen species specifically inactivate the exonuclease active site of gene 5 protein; (4) to determine the location of the metal binding site, the identity and location of the damaged residues, and the locations of both the exonuclease and polymerase active sites; (5) to genetically modify the gene 5 protein and thioredoxin, to eliminate undesirable properties such as exonuclease activity and to enhance other properties; and (6) to take advantage of other replication proteins of phage $\mathrm{T} 7$ that interact specifically with $\mathrm{T} 7$ DNA polymerase in order to extend its applications to DNA sequencing. 
Sequencing of Linear Molecules

Jaklevic, J.M.; Kolbe, W.F.; Hadeishi, T.

$\$ 69,000$

Instrumentation Division, Lawrence Berkeley Laboratory, Berkeley, CA, 94720. 415-486-5647;FTS 451-5647

This project involves the application of physical analytical methods to the determination of the base sequence of individual DNA molecules. The initial approach employs a low-temperature solid matrix as a means of immobilizing the DNA in order to facilitate manipulation and to enhance the analytical sensitivity for base identification. Sequential base identification will be achieved using an analytical method with a sensitivity appropriate for distinguishing the four base types. The most sensitive methods available involve the use of resonance absorption followed by mass spectroscopic identification. Initial studies will emphasize the low-temperature spectroscopy of DNA and the physical manipulation techniques associated with matrix isolation. Long-term feasibility will depend upon the demonstration of adequate analytical selectivity within the spatial resolution required for single base identification.

Advanced Concepts for Base Sequencing in DNA

Jett, J.H.; Keller, R.A.; Martin, J.C.; Shera, E.B.

$\$ 366,000$

Life Sciences Division, Los Alamos National Laboratory, Los Alamos, NM, 87545. 505-667-3843; FTS 843-3843; 505-667-3018;FTS 843-3018

This project focuses on sequencing the bases in DNA. Emphasis is on sequencing methods that are rapid, require little DNA (in most cases only a single strand), and are capable of sequencing large fragments to reduce or eliminate mapping requirements. We are considering seven techniques: (1) detection of single, fluorescinated bases in a modified flow cytometer as they are sequentially cleaved from a DNA fragment; (2) acoustic microscopy where a resolution of /approximately/2 /angstrom/ appears possible; (3) base-specific probes for an analog of an electron tunneling microscope; (4) $x$-ray microscopy, to maximize the high resolution available with ultrashort wavelength light; (5) neutron microscopy, to maximize the low-energy deposition and short wavelength resolution that result from the use of cold neutrons as a probe; (6) laser desorption and mass spectrometric analysis of an array of sequentially cleaved bases; and (7) flow cytometric analysis of fragments, using a modification of the Hood technique.

\section{Informatics}

Development of VLSI-Based Coprocessor Systems Addressing Pattern-Matching Problems of Nucleic Acid and Protein Sequence Data Hood, L.E.

Cancer Center, California Institute of Technology, Pasadena, CA, 91125. $818-356-6357$

We will develop state-of-the-art software and hardware systems for analyzing molecular biology data. These systems will include graphics-based engineering workstations based on powerful workstation computers augmented with specialized coprocessors designed to enhance the speed of important pattern analysis functions. Development of an alignment coprocessor will be an incremental, evolutionary process. No single chip will appear soon that can 
deal with all the searching functions described above. We foresee a series of hybrid coprocessors incorporating more and more of the needed functionalities in custom-made chips. Our initial aim is to define the range of alignment procedures and parameters that should utltimately be incorporated into the coprocessor and to prioritize these according to importance and feasibility of algorithmic schemes within the context of current chip technology. Initial effort will involve designing the algorithms and basic chip and printed circuit (PC) board layout for the first system. Chip fabrication should begin by the fourth quarter of the first year. A thorough analysis and design phase is critical for the development of a useful and functional system. A gradual refinement of the initial system and the addition of further functionalities are expected in succeeding years. Novel algorithms and new chip technologies will continue to be evaluated.

\section{Human Genome Information System}

Quong, $C$.

Computer Science Research Department, Lawrence Berkeley Laboratory, 1

Cyclotron Rd., Berkeley, $C A, 94720$.

$415-486-6630$

A three part Human Genome Information Systems Program consisting of systems development, research in data management, and research in data analysis is in progress. In the systems development area, the initial concern is primarily to help acquire and organize the computing resources, both hardware and software, needed for the mapping and cloning activities. In the data management area, we will pursue research and development of new techniques needed for handiing large scale molecular biological data. Existing database technology lacks much of the basic foundations for handling the types and complexity of genomic data. We will investigate methods for describing, storing, indexing and searching partial, ambiguous genetic and physical genomic maps, composite sequence data, homology data, and structural data. Our data analysis efforts are directed initially at using combinatorial algorithm approaches for the development of sequence data analysis tools needed for approximate sequence and pattern matching. Techniques developed here might be incorporated as approximate matching operators in the sequence data manager. Algorithms for fragment overlap detection and map assemble will be investigated. For genomic structure analysis, we will investigate the application of neural networks techniques to recognize structural features in DNA sequences. 
1987 Smal1 Business Innovative Reseach (SBIR) Awards

The SBIR proposals are reviewed and funded through a DOE wide system which is independent of and not competitive with Genome Initiative processes and resources.

Microcomputer-Based DNA Gel Electrophoresis Image Analysis Hozier, J.C.,

Genetics Data Services, Inc., Melbourne, FL, 32901. 305-768-2048

$\$ 37,894$

The project objective is to design and construct a microcomputer-based

instrument for quantitating size distributions of nucleic acids (DNA and RNA) separated according to size by gel electrophoresis. Analysis of

fluorescence-stained DNA in gels will begin with the image digitization of the entire gel electrophoretic pattern (or specified portions) so that the relative intensities of fluorescence will be converted directly to image data in computer memory. The image processing system will perform quantitative measurements of the amount of DNA of various sizes, using semiautomated comparison to DNA size standards. Advantages of such a system include (1) flexibility, precision, and reliability in the analysis; (2) acceptance of varying formats for gel electrophoresis; and (3) relatively low cost, because the system is microcomputer-based and can be assembled from stock components. Phase I will focus on investigations of hardware component requirements, assembly of a preliminary hardware design to demonstrate feasibility of the planned configuration for a workable gel-digitization analysis instrument, and initial software development to demonstrate the potential analytical utility of such a system. Phase II will involve construction, thorough testing and validation of the overall hardware system design, and complete generation of a software system for accurate and versatile analysis of DNA size distributions on gels. System design will provide the broadest and most versatile set of DNA analytic functions possible, to accommodate new applications in molecular genetics, gene mapping and sequencing, and genetic toxicology.

An Improved Imaging System Using A CCD Detector to Quantitate Fluorescence from Stained DNA Gels

Myers, S.A., $\$ 49,581$

Instrumentation Development, Inc., Old Greenwich, CT, 06870. 203-637-2010.

The mass of DNA separated by gel electrophoresis can be determined from the intensity of the fluorescence of a DNA-specific dye excited by ultraviolet radiation. Use of photographic film to record the fluorescence limits the accuracy, sensitivity, and dynamic range of the analysis. Alternate scanning systems that directly detect the fluorescence are slow. A new imaging system is planned to measure mass and molecular weight of the sample. Substantial instrumental improvements are possible in fluorescent excitation, optical light collection and imaging, and detector performance. Replacing film with a charge-coupled array detector (CCD) is possible because of recent improvements in CCD performance and the availability of larger arrays at lower cost. Optical properties of the stained DNA/gel system will be measured, which must be well-characterized to design a high-performance imaging system and to estimate its performance accurately. An engineering analysis of a specific design will (1) examine the increased analytical ability to quantitate the mass of DNA in a band through improved fluorescence 
measurement and (2) determine whether the overall measurement time can be significantly shortened by achieving high detector signal-to-noise and by more fully automating the measurement. Phase I will address the feasibility of the new instrumental approach, estimate the limits of performance, and provide a design direction for the prototype hardware to follow in Phase II.

Chemiluminescent Labels for Polynucleotides Electrophoretically Separated in Agarose Gels

Davis, E.M. ,

SymBiotech, Inc., Cheshire, CT, 06410. 203-272-4190

$\$ 500,000^{*}$

The project objective is to continue the Phase I work that demonstrated the feasibility of using chemiluminescence to visualize polynucleotides

electrophoresed in agarose gels. Chemiluminescence is a very powerful labeling method, and under optimal conditions yields detection limits equal to or better than radioisotopic labels. Phase II will focus on improving the detection limits for DNA in electrophoresis gels. The approach is to develop improved ways to decrease background luminescence, to increase the efficiency of biotinylation of DNA, and to explore more powerful luminescent reagents. The ultimate aim is to develop highly sensitive and safe labels that can be detected with inexpensive photography or be recorded, processed, and stored by an electronic digital imaging system. We will also explore the potential of using chemiluminescence for labeling DNA fragments in sequencing gels. We believe that several products will be developed from this work, including a combination electrophoretic device for labeling and photographing luminous DNA. In addition, new reagents for improved luminescence and biotinylation of DNA will be developed for commercialization.

* A phase II award received after successful completion of phase I goals.

\section{Smal1 Business Innovation Research Awards}

A Nove1, Low Cost System for Automated DNA Sequence Reading Vickers, M.G. $\$ 48,321$ BioAutomation Associates, 113 North Spring Mill Road, Villanova, PA, 19085. $215-525-5325$

Two types of instruments are emerging for the automated measurement of DNA. One uses fluorescent labeling and detection in the gel. The other uses radioactive labeling and detection from an autoradiogram. Innovations such as multiplex sequencing suggest that both techniques will be used in years to come for both mapping and sequencing. Instrumentation for the two approaches has been sophisticated and expensive (typically $\$ 90,000$ per instrument). As a result, fewer than one percent of the 10,000 plus sequencing labs worldwide have acquired such instrumentation. Because of high cost, adoption of automated DNA sequencing instrumentation will be slow even though interest is high. This threatens to restrict major sequencing efforts to a few "big science" laboratories, hindering the advancement of the field. The goal of this work is to demonstrate the technological feasibility of scanning instrumentation for automated sequence reading with a cost an order of magnitude lower, and hence more accessible to smaller $1 \mathrm{abs}$. This work 
includes construction of a test fixture and measurments made with it. If successful, these measurements will demonstrate the feasibility of a low cost approach.

The Separation of Large DNA Fragments with Oscillating Magnetic Fields Morris, E.R.

BTX, Inc., 3742 Jewell Street, San Diego, CA, 92109. 619-270-0861

A moving charge in a magnetic field experiences a force (Lorentz force) perpendicular to both the direction of the movement and the magnetic field. Charges subjected to oscillating electric fields and an oscillating perpendicular magnetic field of the same frequency and a predetermined phase relationship will drift unindirectionally. Polarization of biological particles represents moving charges. By applying oscillating crossed electric and magnetic fields (OCEMF), biological particles will drift at a velocity dependent on size and dielectric properties and can be sorted into fractions. We demonstrated this effect on a variety of cells and observed a sensitive dependency of the drift velocity on cell properties. DNA exhibits a large induced dipole moment at low frequencies with a relaxation time dependent on the length of the fragment. We propose to induce DNA fragments to drift in OCEMF. Because the drift direction is "sideways" to the electric field and the velocity depends on the length of the DNA fragment, we can expect better separation of very large DNA fragments (>500 kBases) than with pulsed field gel electrophoresis. The proposed technique is expected to speed up the process of sequencing the human genome.

\section{A DNA/Protein Sequence Analysis Database Accelerator} Alexander, $P$. Numerix Corporation, 20 Ossipee Road, Newton, MA, 02164. 617-964-2500

The rapid growth in size of DNA and protein databases that will accompany the human genome project, is sure to overburden available computer resources. Homology searches, optimal sequence comparisons, and studies of RNA secondary structure are all important molecular biology research topics, requiring the availability of powerful database and computational facilities. This investigation will explore the relationships between popular and emerging computerized sequence analysis algorithms, including DNA/Protein sequence mapping and RNA folding programs, and their fundamental computer architectural requirements. A baseline system, configured from commercial available elements, will be analyzed. If implemented, it would provide 100 MIPS of equivalent processing power to networked research teams at the equivalent cost of a superminicomputer. 
A Real-Time Imaging System for Enhancing DNA Hybridization Technologies Entine, $G$.

Radiation Monitoring Devices Inc., 44 Hunt Street, Watertown, MA, 02172. $617-926-1167$

One of the most active and challenging fields in molecular biology today is the genetic study of eukaryotic systems. The preliminary, but often rate limiting steps in this area are the DNA transfer/hybridization technologies associated with gene mapping, and the screening of DNA libraries for gene isolation. These procedures use radiolabeled hybridization techniques and autoradiography to achieve the required detection sensitivity and specificity. The particular part of the process that this proposal addresses is the measurement of the distribution of radioactivity on the transfer membrane. Normally the autoradiographs taken of these membranes require an exposure of 16 to 24 hours. Furthermore, because of the uncertainty in sample activity, the autoradiographic step is often iterated to bracket the exposure times and obtain the maximum image sharpness. We propose to utilize a newly available, position sensitive nuclear detector to generate a real time image of the distribution of the radioactivity on the transfer membrane. Furthermore, we propose to couple this image detection system to a laboratory computer for data storage and analysis. By this approach, not only will the researcher be able to optimize data without suffering the dalays associated with autoradiography, but will also be able to carry out the procedure with a much higher level of confidence. This ability will significantly improve the efficiency of the entire procedure and allow more resources to be directed toward the analytical aspects of the work. 